\title{
Social Support Intervention for Self-Management of Type 2 Diabetes Mellitus: Study Protocol for a Randomized Controlled Trial
}

\author{
Claudia Milena Garizábalo- \\ Dávila (D) \\ Alba Luz Rodríguez- \\ Acelas (D) ${ }^{\prime}$ \\ Rita Mattiello $\mathbb{D}^{2}$ \\ Wilson Cañon- \\ Montañez (D) \\ 'Faculty of Nursing, Universidad de \\ Antioquia, Medellín, Colombia; ${ }^{2}$ School \\ of Medicine, Pontifícia Universidade \\ Católica do Rio Grande do Sul, Porto \\ Alegre, Brazil
}

\begin{abstract}
Background: Social support interventions seek to improve the self-management of type 2 diabetes mellitus (T2DM). They provide an opportunity to generate greater social value in nursing care and achieve preventive health behaviors.

Methods: A randomized controlled trial will be employed, covering 94 adults diagnosed with T2DM who attend outpatient diabetes consultations. Using 1:1 random allocation, 47 patients will be assigned to the social support intervention group and 47 will be assigned to the usual care group. The participants will be assessed using the following Nursing Outcomes Classification (NOC) labels: self-management: diabetes (1619); and social support (1504). The study participants will receive four individualized fortnightly sessions of social support, lasting 40 minutes each, and will be followed up on for three months afterwards. Results: The participants in the social support intervention group are expected to achieve greater self-management of T2DM than the participants in the usual care group.

Discussion: Testing a social support intervention provided to adults by nurses for selfmanagement of T2DM is a novel approach based on disciplinary theoretical support. It is consistent with the new paradigms, providing opportunities for comprehensive care and enabling those who live with diabetes to be more autonomous and empowered and to enjoy better health outcomes and a better quality of life.
\end{abstract}

Keywords: self-management, social support, diabetes mellitus, type 2, nursing, protocol

\section{Introduction}

Diabetes is one of the biggest public health problems and has come to be considered a global health emergency of the 21 st century. The International Diabetes Federation estimates that 463 million adults worldwide are currently affected by this condition, with a projected increase of 700 million by $2045 .{ }^{1}$ Similarly, cases of type 2 diabetes mellitus (T2DM), which represents $90 \%$ of diabetes cases worldwide, are increasing globally. Between 1990 and 2017, the age-standardized rates increased from 228.5 to 279.1 in terms of incidence and from 4.57 to 5.72 in terms of prevalence. ${ }^{2}$

Various studies show that self-management achieves effective management of blood glucose levels and reduces the risks of complications. It allows for the modification of dietary and physical activity behaviors; and learning strategies to prevent or respond effectively to hypoglycemia and hyperglycemia. ${ }^{3-7}$

People with diabetes mellitus have a high burden of disease, associated with multiple physical, psychological, and social alterations, which influence the
Correspondence: Wilson CañonMontañez

Faculty of Nursing, Universidad de Antioquia, Cl. 64 \#53-09, Medellín, 050010 , Colombia

Tel +5742196300

Email wilson.canon@udea.edu.co 
patient's ability to control their health, to engage in decision making and problem solving, and to cope with their condition. ${ }^{8,9}$ However, the current interventions that seek to improve self-management in adults with T2DM focus on addressing physiological variables and typical selfmanagement behaviors such as healthy eating and physical activity; but there are limited studies on interventions that address psychosocial variables such as social support. ${ }^{10-16}$

Recent studies suggest social support as a complementary intervention that can contribute to improving clinical outcomes (healthy lifestyle behaviors and physical activity) ${ }^{9,17,18}$ and quality of life at a lower cost. However, there is still a scarcity of randomized controlled trials that evaluate the efficacy of social support in controlling T2DM.

This study is based on Individual and Family SelfManagement Theory (IFSMT), ${ }^{19}$ which covers three dimensions that seek to improve health outcomes in people with chronic diseases: the context, the process, and the results. The study takes into account the following key aspects of the theory for people with T2DM to remain in control: (a) knowledge and beliefs; (b) self-management skills; and (c) social support (Figure 1).

Knowledge refers to objective information specific to the condition; and beliefs are understood as personal perceptions about the specific health condition or health behavior. An improvement in knowledge and beliefs results in a greater understanding of T2DM. Self-management skills are individuals' abilities to carry out activities correctly and easily, allowing them to self-manage their T2DM. Social support consists of the emotional, instrumental, and informative support provided to people living with diabetes, with the explicit objective of helping or facilitating their participation in self-management behaviors. In the specific case of this study, this will be provided by a nurse. It is expected that by the end of the intervention the participants will show improved self-management behavior and be more autonomous and empowered in their health processes and have better control over their disease.

\begin{tabular}{|c|c|c|}
\hline Risk and protective factors & $\begin{array}{c}\text { Nursing intervention for the } \\
\text { individual with type } 2 \\
\text { diabetes mellitus }\end{array}$ & Results \\
\hline $\begin{array}{l}\text { Personal characteristics I } \\
\text { lifestyles } \\
\text {-Knowledge } \\
\text {-Beliefs } \\
\text {-Life patterns } \\
\text { Health condition } \\
\text {-Comorbidities } \\
\text {-Severity of the disease } \\
\text {-Symptoms } \\
\text { Means } \\
\text {-Financial } \\
\text {-Team } \\
\text { Environmental } \\
\text { characteristics } \\
\text {-Home } \\
\text {-Job } \\
\text {-Community } \\
\text { Health care system } \\
\text {-Access }\end{array}$ & $\begin{array}{l}\text { - Knowledge } \\
\text {-Self-management skills } \\
\text {-Social support }\end{array}$ & $\begin{array}{l}\text { Proximal } \\
\text {-Self-management } \\
\text { behaviors: } \\
\text { *Healthy nutrition } \\
\text { *Physical exercise } \\
\text { *Glucose monitoring } \\
\text { *Symptom management } \\
\text {-Social support }\end{array}$ \\
\hline
\end{tabular}

Figure I Theoretical model adapted for the study. This model contemplates self-management as the "ability of the individual, in conjunction with the family, the community, and health professionals, to manage symptoms, treatments, changes in lifestyle, and psychosocial, cultural, and spiritual consequences of health conditions." The study considers knowledge, self-management skills, and social support as active principles of the intervention. 
The objective of this study is to assess the efficacy of an adult social support intervention for self-management of T2DM. We hypothesize that the participants in the social support intervention group will achieve better selfmanagement of T2DM than the participants in the usual care group.

\section{Methods}

\section{Study Design}

A randomized controlled trial is used that follows the guidelines of SPIRIT (Standard Protocol Items: Recommendations for Interventional Trials). ${ }^{20}$ The study protocol has been registered at ClinicalTrials.gov (NCT04491253).

\section{Randomization and Blinding}

Simple randomization will be performed in groups with equal sample sizes, using the Epidat 4.2 software. The randomization and allocation concealment will be carried out by the principal investigator, who will not participate in the intervention or in the assessment of the outcomes. Adults with T2DM will be assigned using 1:1 random allocation to the social support intervention group and to the usual care group. Regarding the blinding, the evaluators will not know to which group (intervention or control) the people with T2DM belong. Similarly, the nurse in charge of carrying out the intervention will be blinded regarding the initial and post-intervention NOC results self-management: diabetes (1619) and social support $(1504)^{21}$ - in order to have no influence on evaluating the outcome variables.

\section{Participants, Setting, and Sample}

The study participants are adults diagnosed with T2DM who attend outpatient diabetes consultations. The participants will be recruited daily from day one until the sample is completed, at a health center in Barranquilla, Colombia. A sample size of $n=94$ participants was calculated, 47 for the support intervention group and 47 for the usual care group, according to the following parameters: effect size, an expected difference of 0.5 in the NOC results between both groups, $90 \%$ statistical power, a $5 \%$ alpha type error, a 0.9 standard deviation in the outcome scores, an average correlation between the first and second evaluation of 0.3 , a 1:1 intervention/control ratio, and $20 \%$ adjustment for losses.

\section{Procedures}

\section{Eligibility Criteria}

The eligibility criteria include: (a) adults over 18 years of age diagnosed with T2DM, attending outpatient diabetes consultations at a health center; (b) people with a score > 30 in the mini mental cognitive test; ${ }^{22}$ (c) people who do not report comorbidities that limit receiving the intervention (for example: hearing loss, deafness, Alzheimer's disease); (d) an initial score of less than or equal to 3 in the NOC results: self-management: diabetes (1619); and social support (1504). ${ }^{21}$

\section{The Study Intervention}

The present study proposes a social support intervention for adults with T2DM that has a theoretical basis and is supported by the scientific literature. The intervention combines three active principles that influence the selfmanagement of T2DM: knowledge, self-management skills, and social support.

The intervention comprises four social support sessions. The first session provides informational support, including the generalities of T2DM. The second session also provides informational support, this time on healthy eating and physical activity. The third session offers instrumental support, with content on safe care, glucose monitoring, medication management, symptom management, and foot care. The last session gives emotional support, addressing issues such as emotions, anguish, stress, communication and decision-making, trust, empowerment, and support groups and networks. ${ }^{8}$ This is provided in person to adults with T2DM by a nurse trained as a diabetes educator (Table 1).

Four individualized intervention sessions lasting 40 minutes each will be scheduled and carried out in the outpatient diabetes consultations at a health center in Barranquilla, Colombia, 0, 15, 30, and 45 days from the start of the intervention (Table 2). Appointments will be scheduled on different dates and times, thus avoiding encounters between the groups.

\section{Intervention Fidelity}

In order to determine the degree to which an intervention is implemented, the fidelity of the "social support intervention in adults for self-management of T2DM" is described in Table 3. This was carried out taking into account the methodological strategies used to monitor and improve the reliability and validity of the behavioral interventions, such as the delivery, reception, and 
Table I Content of the Social Support Intervention for Self-Management of Type 2 Diabetes Mellitus

\begin{tabular}{|l|l|}
\hline Active Principles of the Intervention & \multicolumn{1}{|c|}{ Knowledge-Self-Management Skills - Social Support } \\
\hline $\begin{array}{l}\text { Session I } \\
\text { Type of social support: } \\
\text { Informational. }\end{array}$ & $\begin{array}{l}\text { Topic: Overview of diabetes } \\
\text { What is diabetes and what is being diabetic like? }\end{array}$ \\
\hline $\begin{array}{l}\text { Session } 2 \\
\text { Type of social support: Instrumental and } \\
\text { informational. }\end{array}$ & $\begin{array}{l}\text { Topic: Healthy eating and physical activity } \\
\text { What strategies should I develop to improve my diet and make it healthier? } \\
\text { How can I organize my life more easily to include more physical activity? }\end{array}$ \\
\hline $\begin{array}{l}\text { Session 3 } \\
\text { Type of social support: } \\
\text { Instrumental. }\end{array}$ & $\begin{array}{l}\text { Topic: Safe care } \\
\text {-Glucose monitoring skills; medication management; symptom management; foot care. }\end{array}$ \\
\hline $\begin{array}{l}\text { Session 4 } \\
\text { Type of social support: } \\
\text { Emotional. }\end{array}$ & $\begin{array}{l}\text { Topic: Emotional support } \\
\text {-Emotions, anguish, and stress; communication and decision making; empowerment and confidence; } \\
\text { support groups and networks. }\end{array}$ \\
\hline
\end{tabular}

fulfillment of the intervention (Table 3); as recommended by the Fidelity Treatment Working Group of the Behavior Change Consortium of the National Institutes of Health. ${ }^{23}$

\section{Data Collection and Measures}

The evaluators will be previously trained and blinded to the intervention strategies. They will evaluate the participants using the NOC results: self-management: diabetes (1619); and social support (1504). This will take place before starting the intervention to provide a baseline, at the end of the intervention, and three months after the end of the four intervention sessions. The nurse who will perform the intervention will have no influence in assessing the outcomes.
At the end of the investigation, printed material will be given to the participants as a form of thanking them for their participation in the study. A study-specific demographic and health history form will be completed at the beginning of the study, which will include variables such as age, sex, marital status, occupation, socioeconomic level, place of residence, social security in health, time since T2DM diagnosis, and glycosylated hemoglobin value.

For the cognitive assessment or examination of the mental state of the study participants, the mini mental test will be used. This instrument is free to use and is composed of 30 dichotomous items that evaluate six cognitive processes: temporal orientation, spatial orientation,

Table 2 Specific Elements and Characteristics of the Intervention

\begin{tabular}{|l|l|}
\hline Name of the Intervention & Social Support Intervention for Self-Management of Type 2 Diabetes Mellitus \\
\hline Session & $\begin{array}{l}-4 \text { sessions } \\
-1 \text { session every I5 days during a period of } 2 \text { months } \\
-2 \text { follow-ups per month for } 2 \text { months after the intervention }\end{array}$ \\
\hline Duration & 40 minutes \\
\hline Delivery strategies & $\begin{array}{l}\text {-Individualized } \\
- \text { Face to face } \\
- \text { Use of educational material (slides and interactive booklet) } \\
\text { Text messaging }\end{array}$ \\
\hline Place of delivery & Nursing consultations (external consultations) at a Health \\
\hline Receiver & Adults with type 2 diabetes mellitus that fulfill the inclusion criteria \\
\hline Supplier & Nursing professional trained in the management of type 2 diabetes mellitus \\
\hline
\end{tabular}


Table 3 Fidelity of the Intervention

\begin{tabular}{|c|c|}
\hline Type of Fidelity in the Intervention & Strategies to Improve the Delivery of the Intervention \\
\hline $\begin{array}{l}\text { Delivery of the intervention Was treatment delivered as } \\
\text { planned? } \\
\text { The intervention is expected to be delivered by a nursing professional } \\
\text { specifically hired for this and who will not be part of the group of } \\
\text { researchers, nor have a working relationship with the institution where } \\
\text { the study will be developed. They will be blinded regarding the initial and } \\
\text { post-intervention results for the nursing results labels, in order to } \\
\text { control the information bias. }\end{array}$ & $\begin{array}{l}\text { - Trained intervention provider. } \\
\text { - Prepare a protocol manual. } \\
\text { - Comply with the intervention protocol. } \\
\text { - Design a checklist. } \\
\text { - Observe an intervention session. }\end{array}$ \\
\hline $\begin{array}{l}\text { Receipt of the intervention } \\
\text { Did the participants demonstrate knowledge and the ability to } \\
\text { use the treatment? } \\
\text { Standardize the processes that control and improve the ability of } \\
\text { participants to understand and perform the behaviors taught in the } \\
\text { intervention. }\end{array}$ & $\begin{array}{l}\text { To ensure the participant's understanding of the content of the } \\
\text { intervention provided, the following will be sought: } \\
\text { Evaluate whether the participant can perform behavioral skills by having } \\
\text { them demonstrate the skills and carrying out an evaluation after each } \\
\text { session. }\end{array}$ \\
\hline $\begin{array}{l}\text { Compliance with treatment } \\
\text { Was the participant able to apply the skills learned during the } \\
\text { treatment to their daily life? } \\
\text { This is the process used to monitor and evaluate the ability of the } \\
\text { participants to perform behavioral skills related to the intervention. }\end{array}$ & $\begin{array}{l}\text { The following will be used to ensure the participatory use of cognitive } \\
\text { and behavioral skills: } \\
\text { - Printed material will be provided. } \\
\text { - Follow-ups will be carried out through discussions with the } \\
\text { participants. } \\
\text { - Participant interviews at the end of the intervention. } \\
\text { - Self-monitoring. } \\
\text { - Follow-up through reminders. } \\
\text { - Measurement by nursing results. }\end{array}$ \\
\hline
\end{tabular}

fixation memory, evocation memory, attention and calculation, and language. ${ }^{22}$

\section{Outcomes}

Two outcomes (self-management and social support) will be assessed. These are part of the NOC classification, ${ }^{21}$ allowing the effects of nursing interventions to be evaluated through a comprehensive and standardized classification of the consequences and responses to nursing activities.

\section{Self-Management}

The NOC result self-management: diabetes (1619) will be used, defined as "personal actions to manage diabetes, its treatment, and to avoid complications," composed of 44 indicators, evaluated on a Likert scale ranging from 1 to 5 ( 1 = never demonstrated, $2=$ rarely demonstrated, $3=$ sometimes demonstrated, $4=$ frequently demonstrated, 5 = always demonstrated). This NOC label belongs to the knowledge and health behavior domain of the health management class, and was last revised in $2013 .^{21}$

\section{Social Support}

The NOC result social support (1504) will also be used, defined as "reliable help from others," composed of 12 indicators, evaluated on a Likert scale ranging from 1 to 5 ( 1 = never demonstrated, $2=$ rarely demonstrated, $3=$ sometimes demonstrated, $4=$ frequently demonstrated, $5=$ always demonstrated). This NOC label belongs to the psychosocial health domain of the social interaction class and was last revised in $2008 .^{21}$

\section{Follow-Up}

The participants will be followed up on for three months, in order to evaluate the effect of the intervention over time.

\section{Data Analysis}

The statistical analysis procedures will be performed in Stata 16.0 by the principal investigator, who will be blind regarding the participant groups.

The principles of intention-to-treat analysis will be followed in order to preserve the sense of randomization. The qualitative variables will be presented as absolute and relative. The quantitative variables with a normal 
distribution will be described as mean and standard deviation, and those that do not present a symmetric distribution will be described as median and interquartile range. To assess the distribution of the quantitative variables, the Kolmogorov-Smirnov test will be used. The variables will be compared between the (intervention and control) groups using the Student's t, chi-squared, or Fisher's exact tests. In order to evaluate the effect of the intervention on the outcomes of the study, an analysis of covariance will be performed.

\section{Ethical Issues}

This trial will be conducted in accordance with the Declaration of Helsinki. Ethics approval was granted by the Research Ethics Committee (REC) of the Faculty of Nursing of the University of Antioquia (REC protocol \# 2020-04). Individual participants will confirm their voluntary participation by providing written informed consent prior to enrollment in the study. The protection and confidentiality of personal data will be guaranteed to the participants. If the proposed intervention shows a positive effect in relation to the studied outcomes, the intervention will also be offered at the end of the study to the participants in the control group.

\section{Results}

The participants in the social support intervention group are expected to achieve greater self-management of T2DM than the participants in the usual care group, presenting improvements in behaviors such as physical activity, healthy eating, symptom management and treatment, as well as an increase in social support. ${ }^{24}$

\section{Discussion}

The study provides an opportunity to examine the efficacy of an adult social support intervention for the self-management of T2DM in the Colombian population. This study will be one of the first to demonstrate that nursing interventions with a focus on social support in adults with T2DM empower them in their own care, imparting greater knowledge and skills to control their health. The study will show that such interventions give these adults the ability to make informed decisions about their condition and, thus, achieve better health results, a higher quality of life, and a positive impact on the economy of the family and the health system. The results will therefore provide important information for the implementation of more holistic interventions, which include the social component as a key element in achieving self-management of T2DM.

It should be noted that if the social support intervention shows efficacy in improving self-management of T2DM, similar interventions could be applied to other chronic diseases. This study will also advance the science in terms of nursing, by using NOC results. ${ }^{21}$ Future studies could continue investigating the effect of interventions using the nursing taxonomies of diagnoses, outcomes, and interventions.

This study may be faced with some limitations. First, there may be a lack of participation by some patients due to economic and sociocultural differences. This will be minimized by encouraging the patients to remain in the study and showing them its importance for self-management of their disease. Second, the study participants may find it difficult to consistently attend all the intervention sessions. However, to ensure they complete the intervention, the participants will receive a text reminder every week via mobile phone of the next session and be encouraged to complete all sessions.

\section{Disclosure}

The authors report no conflicts of interest in this work.

\section{References}

1. International Diabetes Federation. IDF Diabetes Atlas, 9th edn. Brussels, Belgium; 2019. Available from: https://www.diabetesatlas. org/. Accessed April 01, 2021.

2. Lin X, Xu Y, Pan X, et al. Global, regional, and national burden and trend of diabetes in 195 countries and territories: an analysis from 1990 to 2025. Sci Rep. 2020;10(1):1-11. doi:10.1038/s41598-02071908-9

3. Azami G, Soh KL, Sazlina SG, et al. Effect of a nurse-led diabetes self-management education program on glycosylated hemoglobin among adults with type 2 diabetes. J Diabetes Res. 2018:1-12. doi:10.1155/2018/4930157

4. Chrvala CA, Sherr D, Lipman RD. Diabetes self-management education for adults with type 2 diabetes mellitus: a systematic review of the effect on glycemic control. Patient Educ Couns. 2016;99(6):926-943. doi:10.1016/j.pec.2015.11.003

5. McEwen MM, Pasvogel A, Murdaugh C, Hepworth J. Effects of a family-based diabetes intervention on behavioral and biological outcomes for mexican american adults. Diabetes Educ. 2017;43(3):272285. doi: $10.1177 / 0145721717706031$

6. Pirbaglou M, Katz J, Motamed M, Pludwinski S, Walker K, Ritvo P. Personal health coaching as a type 2 diabetes mellitus self-management strategy: a systematic review and meta-analysis of randomized controlled trials. Am J Health Promot. 2018;32(7):1613-1626. doi:10.1177/0890117118758234

7. Stephani V, Opoku D, Beran D. Self-management of diabetes in SubSaharan Africa: a systematic review. BMC Public Health. 2018;18 (1):1-11. doi:10.1186/s12889-018-6050-0

8. Garizabalo-Dávila CM, Rodríguez-Acelas AL, Cañon-Montañez W. Soporte social enfocado a personas con diabetes: una necesidad desde enfermeria. Rev Cuid. 2019;10(1):e697. doi:10.15649/cuidarte. v10i1.697 
9. Ramkisson S, Pillay BJ, Sibanda W. Social support and coping in adults with type 2 diabetes. Afr J Prim Health Care Fam Med. 2017;9 (1):1-8. doi:10.4102/phcfm.v9i1.1405

10. Bowen ME, Cavanaugh KL, Wolff $\mathrm{K}$, et al. The diabetes nutrition education study randomized controlled trial: a comparative efectiveness study of approaches to nutrition in diabetes self-management education. Patient Educ Couns. 2017;99(8):1368-1376. doi:10.1016/ j.pec.2016.03.017

11. Glasgow RE, Kurz D, King D, et al. Twelve-month outcomes of an Internet-based diabetes self-management support program. Patient Educ Couns. 2012;87(1):81-92. doi:10.1016/j.pec.2011.07.024

12. Moriyama M, Nakano M, Kuroe Y, Nin K, Niitani M, Nakaya T. Efficacy of a selfmanagement education program for people with type 2 diabetes: results of a 12 month trial. Jpn J Nurs Sci. 2009;6 (1):51-63. doi:10.1111/j.1742-7924.2009.00120.x

13. Shaya TF, Chirikov VV, Howard D, et al. Effect of social networks intervention in type 2 diabetes: a partial randomised study. $J$ Epidemiol Community Health. 2014;68(4):326-332. doi:10.1136/ jech-2013-203274

14. Spencer MS, Rosland A-M, Kieffer EC, et al. Effectiveness of a community health worker intervention among african american and latino adults with type 2 diabetes: a randomized controlled trial. $\mathrm{Am} \mathrm{J}$ Public Health. 2011;101(12):2253-2260. doi:10.2105/ AJPH.2010.300106

15. Stopford R, Winkley K, Ismail K. Social support and glycemic control in type 2 diabetes: a systematic review of observational studies. Patient Educ Couns. 2013;93(3):549-558. doi:10.1016/j. pec.2013.08.016

16. Sun AC, Tsoh JY, Saw A, Chan JL, Cheng JW. Effectiveness of a culturally tailored diabetes self-management program for Chinese Americans. Diabetes Educ. 2012;38(5):685-694. doi:10.1177/ 0145721712450922
17. Rosland AM, Piette JD, Lyles CR, et al. Social support and lifestyle vs. Medical diabetes self-management in the diabetes study of Northern California (DISTANCE). Ann Behav Med. 2014;48 (3):438-447. doi:10.1007/s12160-014-9623-x

18. Song Y, Song H, Han H, Park S, Nam SKM. Unmet needs for social support and effects on diabetes self-care activities in Korean Americans with type 2 diabetes. Diabetes Educ. 2012;38:77-85. doi:10.1177/0145721711432456

19. Ryan P, Sawin KJ. The individual and family self-management theory: background and perspectives on context, process, and outcomes. Nurs Outlook. 2009;57(4):217-225.e6. doi:10.1016/j. outlook.2008.10.004

20. Chan AW, Tetzlaff JM, Altman DG, et al. SPIRIT 2013 statement: defining standard protocol items for clinical trials. Ann Intern Med. 2013;158(3):200-207. doi:10.7326/0003-4819-158-3-20130205000583

21. Moorhead S, Johnson M, Mass ML, Swanson E. Nursing Outcomes Classification (NOC). 6th ed. Elsevier; 2018.

22. Rojas-Gualdrón DF, Segura CA, Cardona AD, Segura CÁ, Garzón MO. Análisis rasch del mini mental state examination (MMSE) en adultos mayores de Antioquia, Colombia. CES Psico. 2017;10(2):1727. doi:10.21615/cesp.10.2.2

23. Bellg AJ, Resnick B, Minicucci DS, et al. Enhancing treatment fidelity in health behavior change studies: best practices and recommendations from the NIH Behavior Change Consortium. Health Psychol. 2004;23(5):443-451. doi:10.1037/0278-6133.23.5.443

24. Gallant MP. The influence of social support on chronic illness selfmanagement: a review and directions for research. Heal Educ Behav. 2003;30(2):170-195. doi:10.1177/1090198102251030
Open Access Journal of Clinical Trials

\section{Publish your work in this journal}

The Open Access Journal of Clinical Trials is an international, peerreviewed, open access journal publishing original research, reports, editorials, reviews and commentaries on all aspects of clinical tria design, management, legal, ethical and regulatory issues, case record form design, data collection, quality assurance and data auditing methodologies. The manuscript management system is completely online and includes a very quick and fair peer-review system, which is all easy to use. Visit http://www.dovepress.com/testimonials.php to read real quotes from published authors. 